\title{
Zellulare Nichtlineare Netzwerke: Anwendungen in der Informationstechnologie
}

\section{R. Tetzlaff}

Institut für Angewandte Physik, Johann Wolfgang Goethe-Universität, Frankfurt/Main, Deutschland

Zusammenfassung. Zellulare Nichtlineare ${ }^{1}$ Netzwerke (CNN) wurden 1988 von Chua und Yang (Chua und Yang, 1988) eingeführt. Diese Netzwerke sind dadurch gekennzeichnet, dass eine Zelle, die die kleinste Einheit eines CNN darstellt, nur mit Zellen innerhalb einer bestimmten Umgebung verbunden ist. Üblicherweise sind Art und Stärke der Wechselwirkung zwischen zwei Zellen eines CNN translationsinvariant, d.h. sie hängen nur von der relativen Lage beider Zellen zueinander ab. Im Vordergrund aktueller Arbeiten stehen auf derartigen Netzwerken basierende schaltungstechnische Realisierungen mit bis zu 176x144 Zellen, die eine direkte Verbindung zu zweidimensionalen optischen SensorAnordnungen aufweisen. Über einen separaten Speicherbereich können die Zellkopplungen eines Netzwerks verändert werden, wodurch eine adaptive Verarbeitung von mehrdimensionalen Sensorsignalen ermöglicht wird. Das kürzlich vorgestellte so genannte EyeRis System (Anafocus Ltd.) enthält zusätzlich noch einen Standardprozessor und stellt (bei einer Größe vergleichbar mit der einer Kreditkarte) daher ein vollständiges superschnelles System zur Informationsverarbeitung dar.

In diesem Beitrag sollen, nach einem kurzen Überblick über die Eigenschaften von CNN, aktuelle Realisierungen und exemplarisch eine neuere eigene Anwendung vorgestellt und besprochen werden.

\footnotetext{
Correspondence to: R. Tetzlaff

(r.tetzlaff@iap.uni-frankfurt.de)

${ }^{1}$ Chua (Chua, 1998) schlug vor, ein derartiges Netzwerk entweder als Zellulares Neuronales Netzwerk oder als Zellulares Nichtlineares Netzwerk abhängig von der betrachteten Problemstellung $\mathrm{zu}$ bezeichnen. Aus Gründen der Vereinfachung soll hier nur die zweite Bezeichnungsweise zugrunde gelegt werden.
}

\section{Einleitung}

Beginnend mit der Einführung im Jahr 1988 durch die grundlegenden Veröffentlichungen von Chua und Yang, stehen seitdem insbesondere Anwendungen zellularer Netzwerke im Vordergrund zahlreicher Arbeiten. Durch die mit der Definitionsweise dieser Netzwerke vorausgesetzte nur lokale direkte Verbindung zwischen einzelnen Zellen, sollte die üblicherweise bei schaltungstechnischen Realisierungen neuronaler Netzwerke vorhandene Verknüpfungsproblematik überwunden werden. Als Folge davon bildet seitdem die Realisierung analoger, CNN-basierter Schaltkreise ein zentrales Thema einer großen Anzahl wissenschaftlicher Arbeiten (Liñán et al., 1999a). Allerdings ist die von Chua ursprünglich angegebene Form der Zustandsgleichung

$\dot{x}_{i j}(t)=-x_{i j}(t)+\sum_{k, l \in N_{i j}(r)} a_{k, l} y_{k l}(t)+b_{k, l} u_{k, l}(t)+z_{i j},(1)$

das so genannte Chua-Yang Modell - für eine Übertragung auf Schaltkreise nur bedingt geeignet. In obiger Form bezeichnet $x_{i j}(t)$ den Zustand einer Zelle $C_{i j}, y_{i j}(t)$ die Ausgangsaktivität und $u_{i j}(t)$ die Eingangswerte. Die Strenge der Rückkopplung und die Gewichtung der Eingangswerte kann mit Hilfe der konstanten reellen Koeffizienten $a_{i-k, j-l}$ und $b_{i-k, j-l}$ eingestellt werden. Die Summation erfolgt in der Zustandsgleichung für die Summationsindizes $k$ und $l$ in der gemäß

$N_{i j}(r)=\left\{C_{k l}:\right.$

$\max (|k-i|,|l-j|) \leq r, 1 \leq k \leq M, 1 \leq j \leq N\}$

definierten Nachbarschaft der Zelle $C_{i j}$. Im einfachsten Fall, d.h. wenn der so genannte Nachbarschaftsradius $r$ den Wert 1 annimmt, besteht eine direkte Verbindung von $C_{i j}$ nur zu Zellen $C_{k l}$ in der unmittelbaren Umgebung; in den meisten 


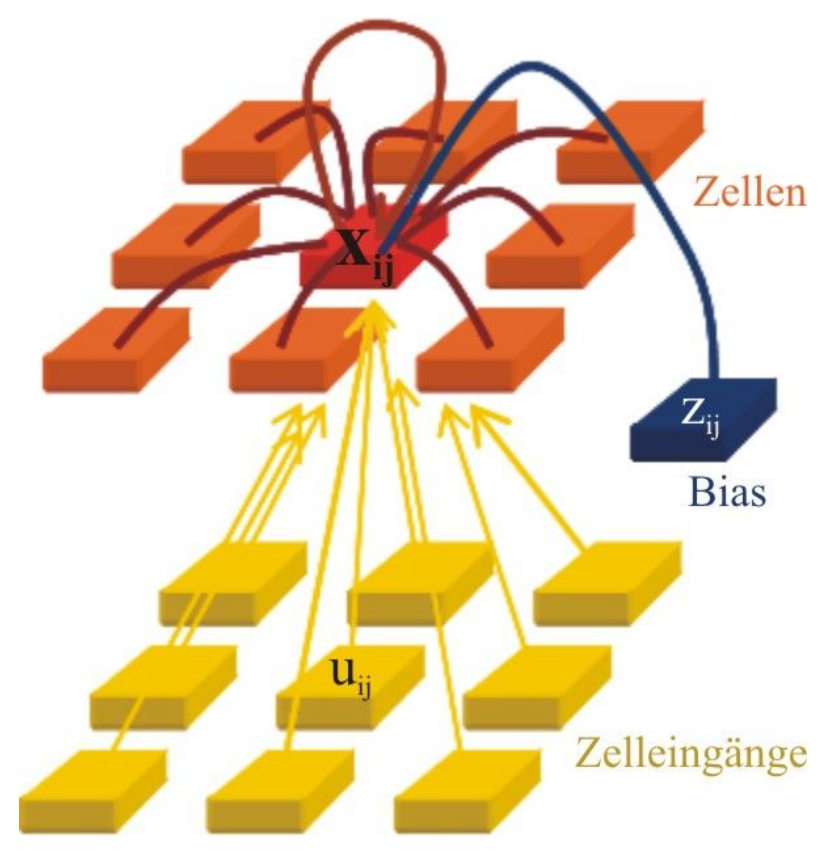

Abb. 1. Die Anordnung von Zellen eines CNN.

Untersuchungen wird ausschließich dieser Fall betrachtet. Auch wird in der Regel die nichtlineare Funktion

$y_{i j}(t)=f\left(x_{i j}(t)\right)=\frac{1}{2}\left(\left|x_{i j}(t)+1\right|-\left|x_{i j}(t)-1\right|\right)$

zur Bestimmung der Ausgangsaktivität $y_{i j}(t)$ anhand des Zellzustands $x_{i j}(t)$ herangezogen, obwohl die Definitionsweise auch andere Abbildungen zulässt. An dieser Stelle sei darauf hingewiesen, dass, wie in den meisten Untersuchungen, in Gl. (1) bereits angenommen wurde, dass die Wechselwirkungen zwischen einzelnen Zellen invariant gegenüber einer Translation im Netzwerk sind. Die Gewichtsfaktoren $a_{i-k, j-l}$ und $b_{i-k, j-l}$ sind daher nur abhängig von den Differenzen der Indizes, welche die Positionen der wechselwirkenden Zellen $C_{i j}$ und $C_{k l}$ im Netzwerk festlegen und können daher auch in Matrixschreibweise als so genannte Templates angegeben werden. Zur Verdeutlichung ist eine Anordnung von Zellen eines CNN in Abb. 1 dargestellt.

Ungünstig für eine schaltungstechnische Realisierung ist das Chua-Yang Modell schon aufgrund der Unbeschränktheit des Zellzustandes in Gl. (1). Wenn die Ausgangsaktivität - bei der üblicherweise verwendeten stückweise linearen Ausgangskennlinie - die Sättigung erreicht, d.h. den Wert 1 annimmt, kann der Zellzustand beispielsweise noch weiter anwachsen. Eine Lösung dieser Problematik gelang durch Einführung des gewöhnlich in Schaltkreisen (Liñán et al., 1999b; Espejo, 1995) verwendeten so genannten Full-Range Modells (Rodríguez-Váquez et al., 1993; Espejo et al., 1994, 1996) mit der Zustandsgleichung

$$
\begin{aligned}
& \dot{x}_{i j}(t)= \\
& -x_{i j}(t)-g\left(x_{i j}(t)\right)+\sum_{k, l \in N_{i j}(r)} a_{k, l} y_{k l}(t)+b_{k, l} u_{k, l}(t)+z_{i j}(4)
\end{aligned}
$$

mit

$$
\begin{aligned}
g\left(x_{i j}(t)\right) & =m\left(x_{i j}(t)-f\left(x_{i j}(t)\right)\right) \\
& =\left\{\begin{array}{cc}
m\left(x_{i j}(t)+1\right) & x_{i j}(t)<-1 \\
0 & \left|x_{i j}(t)\right| \leq 1 \\
m\left(x_{i j}(t)-1\right) & x_{i j}(t)>1 .
\end{array}\right.
\end{aligned}
$$

Bei einem hinreichend großen Wert $m$ führt diese Abbildung dazu, dass der Zellzustand nahezu nur Werte in dem Intervall $[-1,1]$ annimmt. Die ursprüngliche Definitionsweise führte jedoch auch zu Problemen bei der Untersuchung nichtlinearer Phänomene - z.B. bei der Modellierung nichtlinearer Systeme (Solitonen, solitäre Wellen) oder bei der Darstellung von nichtlinearen Reaktions-Diffusionsgleichungen (Murray, 1998) - und wurde daher im Laufe der Zeit von Chua und Roska in verschiedenen Publikationen (Roska und Chua, 1992; Chua et al., 1995) in wachsendem Maße verallgemeinert. Diese Vorschläge führten letztlich zu der ziemlich weit gefassten Definition (Chua, 1998):

Ein CNN ist eine räumliche Anordnung lokal gekoppelter Zellen, von der jede ein dynamisches System darstellt, das über einen Eingang, einen Ausgang und einen Zustand verfügt, der sich nach vorgeschriebenen Gesetzen entwickelt.

Seit der Einführung von CNN wurden diese Netzwerke in zahlreichen Untersuchungen studiert; dies wird durch weit mehr als 1000 Publikationen nachhaltig belegt. Zusätzlich zu den grundlegenden Arbeiten von Chua und Roska (Chua und Roska, 1993; Chua, 1999; Chua und Roska, 2005) wurde das dynamische Verhalten von CNN auch in den Beiträgen von Gilli et al. (Civalleri, 1993; Gilli et al., 2002; Biey et al., 2003) und Forti (Di Marco et al., 2000, 2002) untersucht. In dieser Hinsicht sind auch die Arbeiten von Tavsanoglu und Arik (Joy und Tavsanoglu, 1998; Arik und Tavsanoglu, 2000) und Vandewalle (Suykens et al., 1997; De Souza et al., 2003) zu nennen. In eigenen Untersuchungen stehen Verfahren zur Identifikation komplexer Systeme durch CNN im Vordergrund, dies gilt insbesondere für neuronale biologische Netzwerke (Gollas und Tetzlaff, 2005a,b) und für solche, die eine Ausbreitung nichtlinearer Wellen wiedergeben - z.B. solitäre Wellen oder Solitonen (Puffer et al., 1996b; Loncar und Tetzlaff, 2000; Tetzlaff, 2003). Des weiteren bilden Methoden zur Simulation und Analyse von CNN (Tetzlaff et al., 1997, 1999a) und die Entwicklung von Verfahren zur Vorhersage und Verhinderung epileptischer Anfälle (Tetzlaff et al., 1999b; Tetzlaff und Kunz, 2001; Kunz und Tetzlaff, 2003; Tetzlaff et al., 2005; Gollas et al., 2004) einen wichtigen Teil eigener Untersuchungen. Durch biologische Systeme motivierte Methoden zur Informationsverarbeitung oder zur Generation von Schrittmustern intelligenter Laufroboter werden von Roska (Rekeczky et al., 2000; Bálya et al., 2002, 


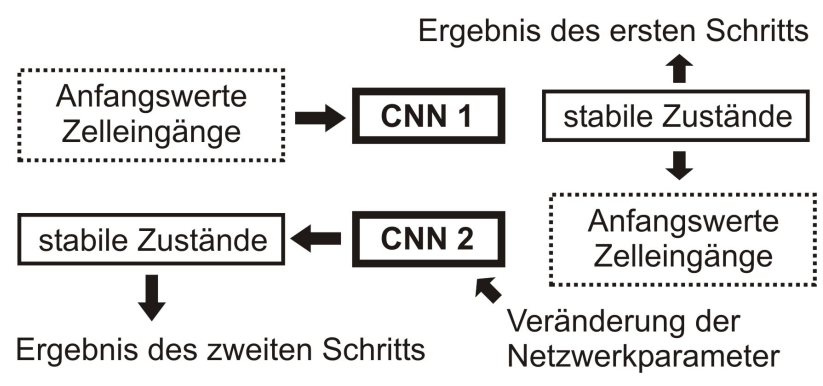

Abb. 2. Einzelne Verarbeitungsschritte eines CNN-Programms.

2004; Werblin und Roska, 2004) und Arena et al. (Arena et al., 2002, 2003; Adamatzky et al., 2004) untersucht. Schließlich werden in zahlreichen Arbeiten CNN-basierte Methoden zur optischen Signalverarbeitung (Crounse und Chua, 1995a; Brendel und Roska, 2002; Feiden und Tetzlaff, 2002, 2003) betrachtet und zur Lösung von Problemen in der Praxis eingesetzt. Eine wichtige Rolle spielen dabei programmierbare Realisierungen, so genannte Cellular Wave Computer, die im folgenden Abschnitt näher betrachtet werden. Alle Veröffentlichungen überschauend kann festgehalten werden, dass viele Untersuchungen interdisziplinär erfolgen. Dabei sollen häufig Phänomene komplexer Systeme zur Lösung einer bestimmten Problematik hinführen.

\section{Zellulare Rechnerstrukturen als intelligente Sensoren}

Stimuliert durch den Nachweis von Chua und Roska (Crounse und Chua, 1995b), d.h. dass mit einer neuartigen Rechnerstruktur, einer so genannten CNN-Universal Machine (CNNUM) (Roska und Chua, 1993), das Game of Life (Berlekamp et al., 1982) implementiert werden kann, fanden umfangreiche Untersuchungen zur Realisierung derartiger Strukturen (Espejo, 1995; Domíniguez-Castro et al., 1994; Liñán et al., 2002; Cruz und Chua, 1994) statt. Einem CNN-UM können demzufolge universelle Eigenschaften zugeordnet werden; es besteht im Wesentlichen aus einem CNN ausgestattet mit analogen und digitalen Speichermodulen. Ein darauf implementiertes Programm ist wie in Abb. 2 prinzipiell aus einer Folge von Verarbeitungsschritten aufgebaut, die mit unterschiedlichen Netzwerken erfolgen. Beispielsweise wird in einem ersten Schritt nach Festlegung einer Anfangs- und Randbedingung die dynamische Entwicklung des Netzwerkes zu einem stabilen Gleichgewicht führen; die sich daraus ergebenden Zellausgangsaktivitäten, können nun für ein anderes CNN als Anfangs- oder Eingangswerte herangezogen werden. Die Fortführung dieser in Abb. 2 dargestellten Vorgehensweise resultiert schließlich zu einem CNN Programm, das somit im Wesentlichen aus einer Folge verschiedener Parametersätze von $\mathrm{CNN}$ besteht.

CNN-UM, die in abstrahierter Form als Cellular Wave Computer bezeichnet werden, eröffnen daher neue Möglich-

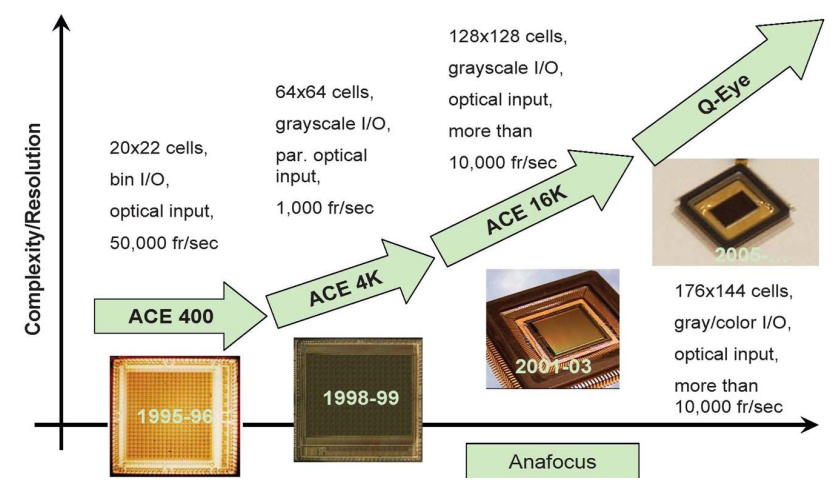

Abb. 3. Übersicht realisierter CVM.

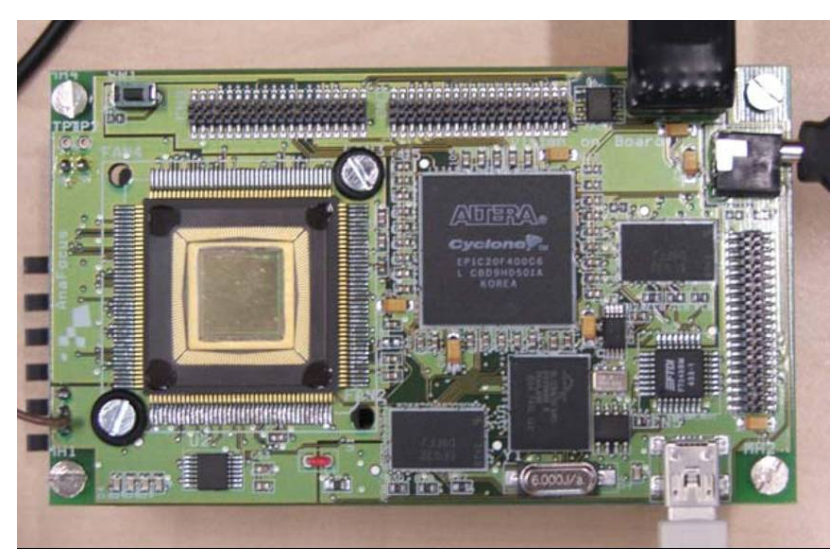

Abb. 4. Das EyeRis-System (Anafocus Ltd).

keiten der Signalverarbeitung. Dabei steht eigentlich die Idee im Vordergrund, das dynamische Verhalten eines derartigen Netzwerks zur Problemlösung einzusetzen; obwohl in einigen Arbeiten nur die enorme, im Bereich von TeraOperationen pro Sekunde liegende Verarbeitungsgeschwindigkeit bei der Anwendung von Standardverfahren nützlich war. Besonders attraktiv für die Informationsverarbeitung sind solche Realisierungen, in denen jede Zelle einen optischen Sensor zur möglichen Messung von Eingangswerten besitzt. Solche so genannten Cellular Visual Microprocessors (CVM) verbinden die Aufnahme visueller Signale mit deren Weiterverarbeitung zu einem Schritt. Abbildung 3 vermittelt eine Übersicht realisierter CVM; in Kürze wird das so genannte Q-Eye Chip (Anafocus Ltd.) erhältlich sein, das über 176x144 Zellen verfügt.

Während ältere Schaltkreise - wie z.B. das ACE400 oder das ACE4K - nur über speziell entwickelte Schnittstellen in Verbindung mit einem PC gesteuert werden konnten, stellen das Bi-i visual system (Zarandy und Rekeczky, 2005) und das Eye-Ris System eigenständige Systeme dar, die über eigene digitale Prozessoren zur Steuerung verfügen. Zur Verdeutlichung zeigt Abb. 4 exemplarisch eine Photographie des Eye-Ris Systems. 


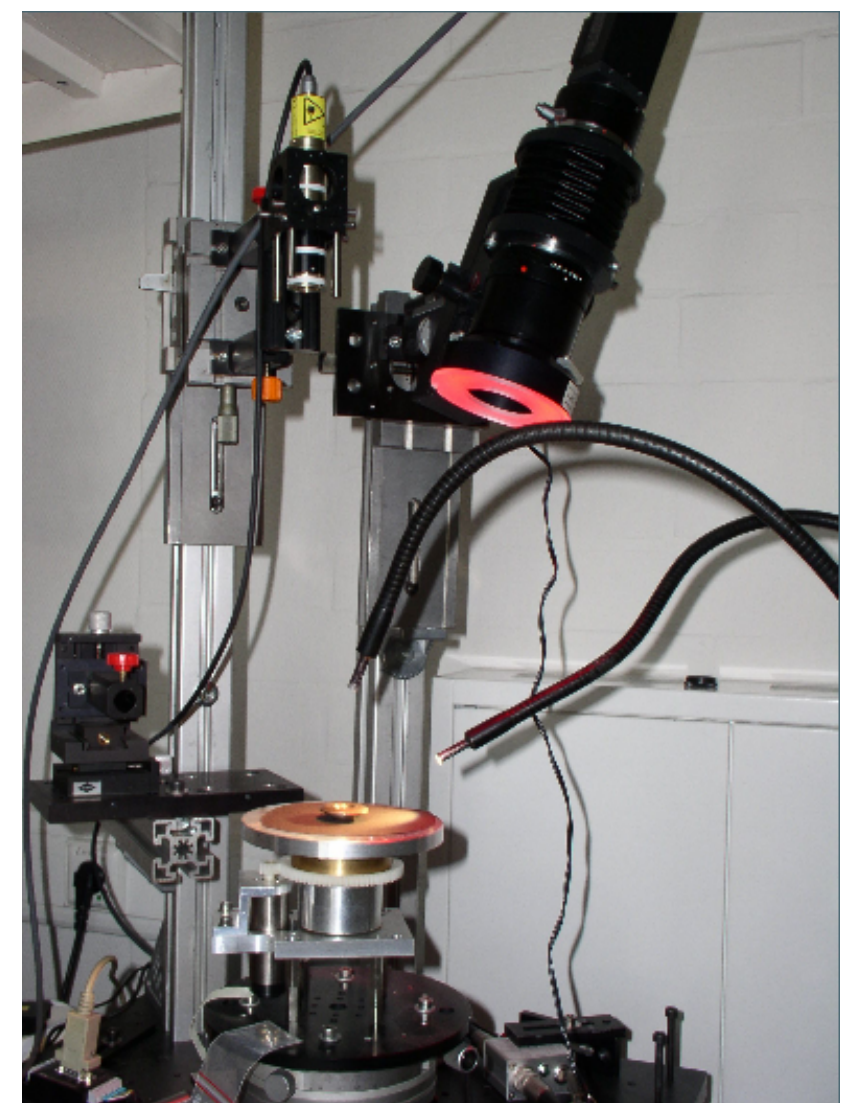

Abb. 5. Der Versuchsaufbau zur visuellen Inspektion von Ventilplatten und Kugellagern.

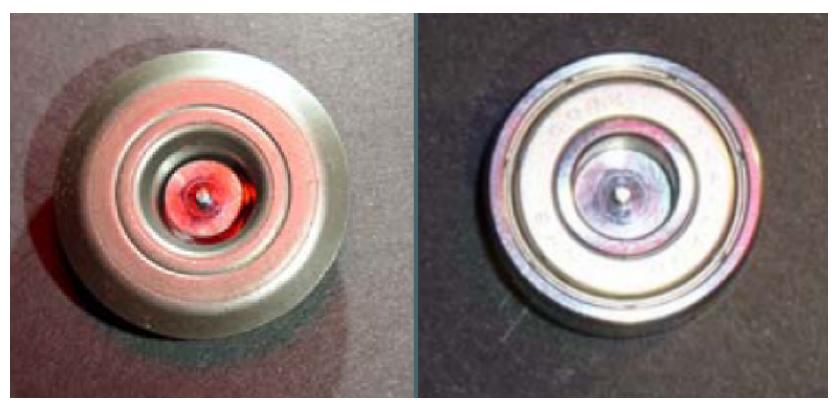

Abb. 6. Beispiel für die verwendeten Kugellager und Ventilplatten.

Alternativ dazu zeigen neuere Veröffentlichungen, dass FPGA basierte Implementierungen (Nagy und Szolgay, 2003; Toledo et al., 2005; Martínez et al., 2005) eine interessante Alternative zu den oben beschriebenen Schaltungen darstellen. Mit derartigen Systemen können vergleichsweise viel größere Netzwerke bei erhöhter Darstellungsgenauigkeit zeiteffizient studiert werden.

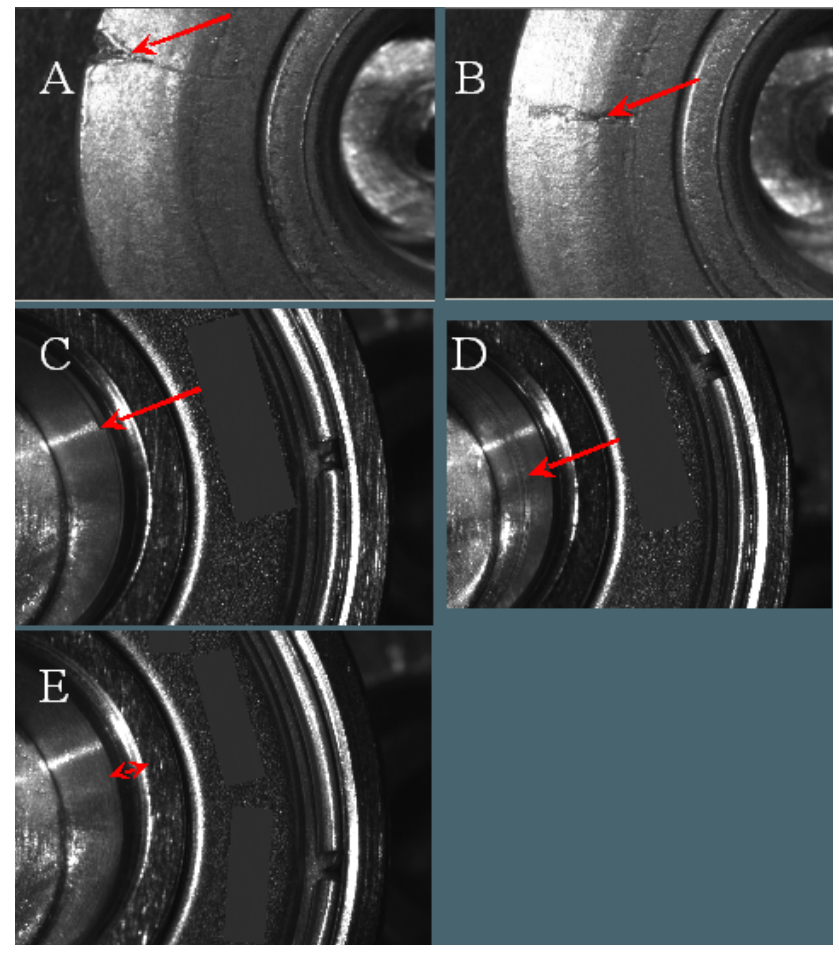

Abb. 7. Beispiele für Beschädigungen der Bauteile. A Ausbruch,B Riss,C Kantengröße,D Kratzer,E Konzentrizität.

\section{CNN zur visuellen Inspektion bei der Herstellung von Metallobjekten}

Aus der beträchtlichen Anzahl von Anwendungen CNNbasierter Methoden in der Informationstechnologie (Rekeczky et al., 1997; Vilarino und Rekeczky, 2004; Saatci und Tavsanoglu, 2002; Koskinen et al., 2002; McRaven et al., 2004; Cserey and Roska, 2003; Kim et al., 2000; Reinisch et al., 2005; Geis et al., 2004; Puffer et al., 1996a; Arena et al., 1999; Gál und Roska, 2000), soll an dieser Stelle exemplarisch über eine eigene Untersuchung (Slavik et al., 2006) kurz berichtet werden, die in Zusammenarbeit mit dem Fraunhofer Institut für Physikalische Messtechnik (IPM) durchgeführt wurde. Im Vordergrund stand dabei eine Methode zur visuellen automatischen Inspektion von Metallobjekten. Üblicherweise werden bei Anwendung einer konventionellen Methode mit einer teilweise hochauflösenden Kamera aufgenommene Bildsequenzen eines beleuchteten Prüflings auf einen PC übertragen und im Rahmen einer Bildanalyse bestimmte Merkmale ermittelt; dabei erfolgen Farbvergleiche und Vollständigkeitskontrollen bis hin zu einer Erfassung 2-3 dimensionaler geometrischer Größen. Unterschiedliche Oberflächenstrukturen und Beleuchtungssituationen schränken jedoch die Leistungsfähigkeit konventioneller Inspektionsmethoden erheblich ein. Im Vergleich dazu ist eine manuelle, durch Menschen durchgeführte Inspektion deutlich robuster und wird häufig sogar zur End- 


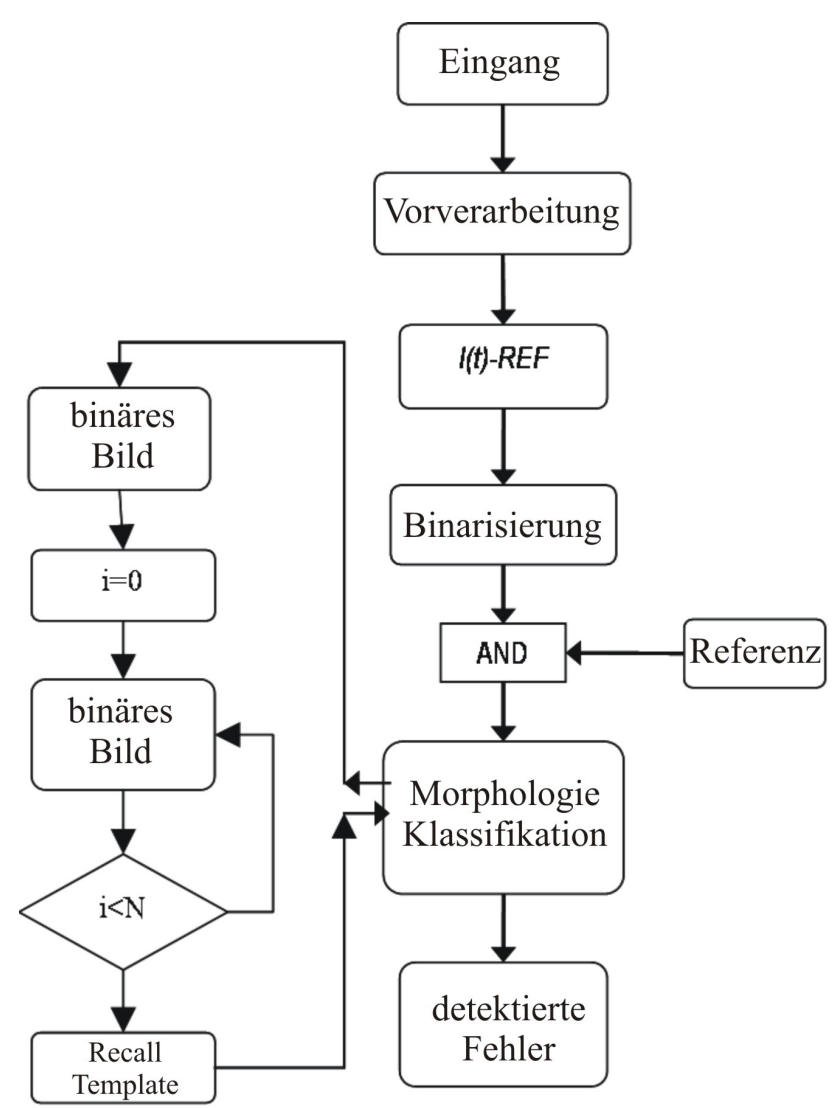

Abb. 8. Darstellung des Verfahrens zur Inspektion von Kugelllagern.

kontrolle eines Produktes in einer ansonsten vollautomatisierten Herstellung eingesetzt. In der im folgenden beschriebenen Studie wurde ein CNN-Verfahren hergeleitet, das der menschlichen Vorgehensweise in einer visuellen Inspektion näher ist, d.h. es wurde eine Fehlererkennung im Rahmen eines Vergleichs zwischen dem fehlerfreien und dem fehlerbehafteten Zustand anhand von unterschiedlichen Aufnahmen des Untersuchungsobjekts vorgenommen. Dazu wurden im IPM mit der in Abb. 5 gezeigten Messapparatur Bildsequenzen aufgenommen, die bestimmte Kugellager und Ventilplatten auf einer rotierenden Platte befestigt zeigen.

Die Beleuchtung der in Abb. 6 dargestellten Untersuchungsgegenstände erfolgte gleichzeitig mit direktem und indirektem Licht. Die dabei verwendeten Kugellager und Ventilplatten waren zum Teil beschädigt.

Als Defektarten lagen für die Ventilplatten Ausbrüche und Risse vor, während für die Kugellager Variationen der Kantengröße, Kratzer und Variationen der Konzentrizität auftraten. Exemplarisch ist in Abb. 7 für jeden derartigen Fall ein Beispiel angegeben.

Die bei den Untersuchungen erhaltenen Ergebnisse belegen, dass mit den implementierten, in Abb. 8 dargestellten CNN Verfahren in nahezu allen Fällen eine zuverlässige Erkennung möglich war. Schwierige Beleuchtungssitua-

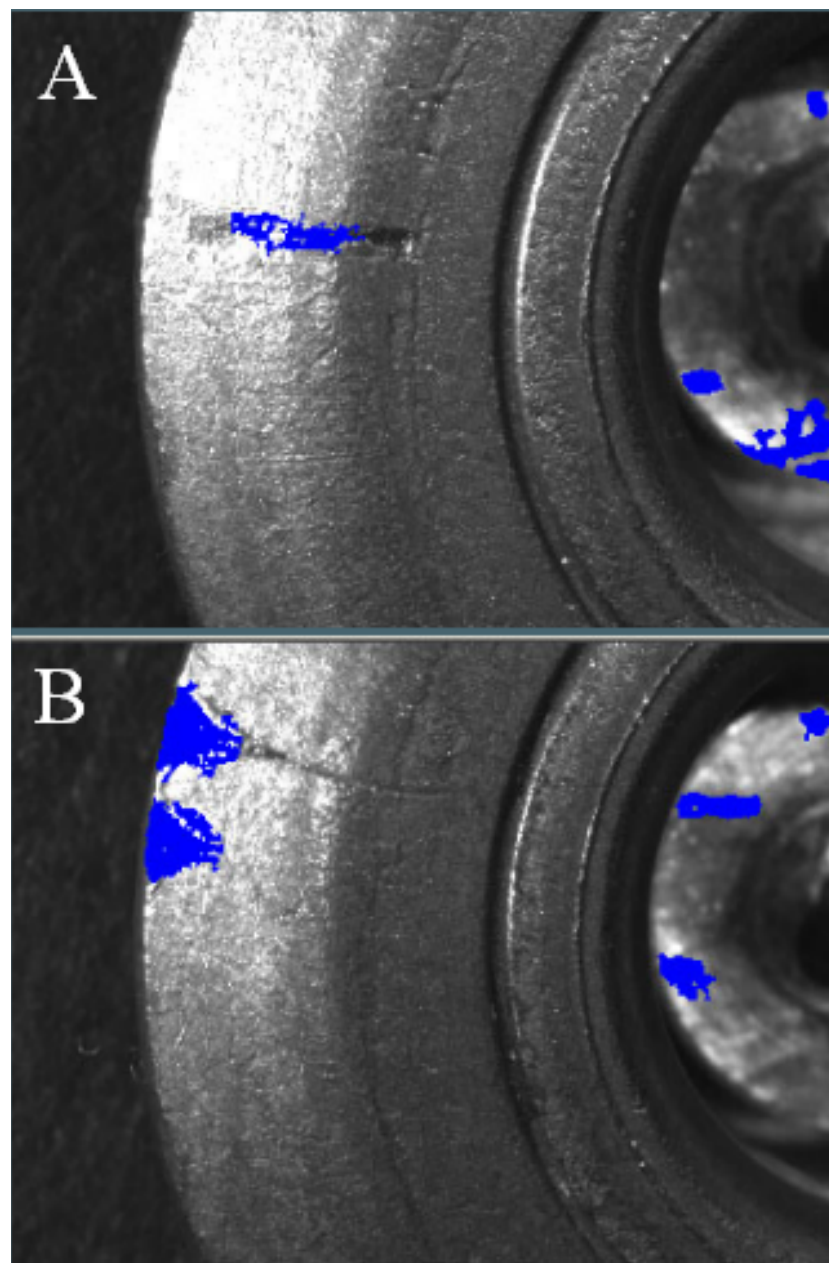

Abb. 9. Exemplarisch dargestellte Ergebnisse. A Riss,B Ausbruch.

tionen unter bestimmten Rotationswinkeln führten bei einer geringen Anzahl von Objekten zu Fehlerkennungen; für einen jeweils anderen Rotationswinkel konnten dann jedoch die Defekte fehlerfrei detektiert werden. Somit erreicht man bei Verwendung von Aufnahmen mit unterschiedlicher Winkeln, d.h. unter geänderten Beleuchtungssituationen, insgesamt eine fehlerfreie Erkennung von Defekten. Die angewendeten Verfahren basieren auf der Beobachtung, dass Defekte ein verändertes Erscheinungsbild des Objektes in Hinblick auf seine Form und der Textur seiner Oberfläche bewirken. Daher erfolgt bei Anwendung dieser Verfahren jeweils nach Verbesserung der Bildqualität im Rahmen einer Vorverarbeitung die Bestimmung eines Differenzbildes; dies sollte dann eventuell vorhandene Defekte des zu untersuchenden Objekts enthalten. Während bei den Ventilplatten sich schon der Vergleich zweier aufeinander folgender Bilder als ausreichend erwies, werden bei den Kugellagern Referenzaufnahmen herangezogen. Im weiteren Verlauf $^{2}$ werden bei

\footnotetext{
${ }^{2}$ Bei den Kugellagern werden vor der Binarisierung, wie in Abb. 8 dargestellt, noch vor der morphologischen Weiterverarbeitung bestimmte für die Detektion relevante Bildbereiche ausgewählt.
} 


\begin{tabular}{cccc}
\hline Sequenz & False negatives & False positives & Wird der Defekt erkannt? \\
\hline Crack 1 & 5 & 0 & Ja \\
Disruption 1 & 1 & 0 & Ja \\
Spots 1 & 0 & 0 & Ja \\
ok 2 & 0 & 0 & Nein \\
ok 3 & 0 & 0 & Nein \\
\hline
\end{tabular}

Tabelle 1. Ergebnisse zur visuellen Inspektion von Ventilplatten mit CNN. Bei der Untersuchung wurden 5 Bildsequenzen mit je 40 Bildern verwendet, die in der ersten Spalte verzeichnet sind. Während die ersten drei Sequenzen unterschiedliche Darstellungen defekter Objekte enthalten, zeigen die zwei anderen Sequenzen fehlerfreie Ventilplatten. In diesen Fällen sind richtigerweise keine Defekte erkannt worden.

\begin{tabular}{cccc}
\hline Sequenz & False negatives & False positives & Wird der Defekt erkannt? \\
\hline IDMark 06 & 1 & 0 & $\mathrm{Ja}$ \\
IDMark 10 & 0 & 0 & $\mathrm{Ja}$ \\
IRCCon 05 & $?^{3}$ & $?^{3}$ & $\mathrm{Ja}$ \\
IDMark 22 & 0 & 0 & $\mathrm{Ja}$ \\
ok 02 & 0 & 0 & Nein \\
ok 03 & 0 & 0 & Nein \\
ok 03a & 0 & 0 & Nein \\
ok b & 0 & 0 & Nein \\
\hline
\end{tabular}

Tabelle 2. Ergebnisse zur visuellen Inspektion von Kugellagern mit CNN. Bei der Untersuchung wurden 8 Bildsequenzen mit je 40 Bildern verwendet, die in der ersten Spalte verzeichnet sind. Während die ersten vier Sequenzen unterschiedliche Darstellungen defekter Objekte enthalten, zeigen die vier anderen Sequenzen fehlerfreie Kugellager. In diesen Fällen sind richtigerweise keine Defekte erkannt worden.

Anwendung beider Verfahren nach einer Binarisierung morphologische Verarbeitungsschritte vorgenommen, d.h. eine mehrmalige Erosion (Chua und Roska, 2002) durchgeführt, die schließlich zur Defekterkennung führt.

Während in Abb. 9 zur Verdeutlichung einzelne Ergebnisse gezeigt sind, vermitteln die Tabellen 1 und 2 einen Überblick über sämtliche Resultate.

\section{Zusammenfassung}

In diesem Beitrag wurde nach einer Einführung in die theoretischen Grundlagen von CNN, eine kurze Übersicht über den momentanen Stand und über die Entwicklung schaltungstechnischer programmierbarer Realisierungen als intelligente Sensoren vermittelt. Derartige Anordnungen bieten neue algorithmische Möglichkeiten und können bei Verwendung ihrer optischen Sensoren zu einer extrem schnellen Bildverarbeitung eingesetzt werden. Schließlich wird als ein aktuelles Beispiel noch die visuelle Inspektion von Metallobjekten vorgestellt. Anhand von Bildsequenzen, die Kugellager und Ventilplatten unter verschiedenen Rotationswinkel zeigen, konnte die Eignung dieser Netzwerke für eine derartige Aufgabenstellung nachgewiesen werden. Eine zuverlässige

\footnotetext{
${ }^{3}$ In dieser Sequenz lag aufgrund der Messung bei verschiedenen Bildern keine eindeutige Fehlerzuordnung vor.
}

Erkennung von Defekten gelang für alle betrachteten Prüfobjekte.

Danksagung. Für die finanzielle und praktische Unterstützung bei der Entwicklung neuer Methoden zur visuellen Inspektion von Metallobjekten sei dem Fraunhofer Institut für Physikalische Messtechnik gedankt.

\section{Literatur}

Adamatzky, A., Arena, P., Basile, A., Carmona-Galán, R., De Lacy Costello, B., Fortuna, L., Frasca, M., and Rodgríguez-Vázquez, A.: Reaction-diffusion navigation robot control: from chemical to VLSI analogic processors, IEEE Transactions on Circuits and Systems-I: Regular Papers, 51, 5, 926-938, 2004.

Anafocus Ltd.: Avd. Isaac Newton s/n, Pabellón de Italia, $7^{a}$ Planta, Ático Parque Tecnológico Isla de la Cartuja 41092 Sevilla, Spain VISION SYSTEMS ON-CHIP - technology, www. anafocus.com.

Arena, P., Fortuna, L., and Branciforte, M.: Reaction-Diffusion CNN Algorithms to Generate and Control Artificial Locomotion, IEEE Trans. on Circuits and Systems I: Special Issue on BioInspired Processors and Cellular Neural Networks for Vision, 46, 253-260, 1999.

Arena, P., Fortuna, L., Frasca, M.: Multi-template approach to realize central pattern generators for artificial locomotion control, International Journal of Circuit Theory and Applications, 30, 441458, 2002. 
Arena, P., Basile, A., Bucolo, M., Fortuna, L., and Virzí, A.: A bioinspired visual feedback locomotion control based on CNN universal machine, Journal of Circuits, Systems, and Computers, 12, 6, 805-823, 2003.

Arik, S. and Tavsanoglu, V.: On the Global Asymptotic Stability of Delayed Cellular Neural Networks, IEEE Trans. on Circuits and Systems I: Fundamental Theory and Applications, 47, 571-574, 2000.

Berlekamp, E. R., Conway, J. H., and Guy, H. K.: Winning Ways for your Mathematical Plays, Academic Press New York, 1982.

Bálya, D., Roska, B., Roska, T., and Werblin, F. S.: A CNN Framework for Modeling Parallel Processing in a Mammalian Retina, International Journal of Circuit Theory and Applications, 30, 363-393, 2002.

Bálya, D., Petrás, I., Roska, T., Carmona, R., and RodríguezVázquez, A.: Implementing the Multi-Layer Retinal Model on the Complex-Cell Stored CNN-UM Chip Prototype, International Journal of Bifurcation and Chaos, World Scientific Publishing Company, ISSN 0218-1274, 14, 427-451, 2004.

Biey, M., Checco, P., and Gilli, M.: Bifurcations and chaos in cellular neural networks, Journal of Circuits, Systems and Computers, 12, 4, 417-433, 2003.

Brendel, M. and Roska, T.: Adaptive Image Sensing and Enhancement Using the Cellular Neural Network Universal Machine, International Journal of Circuit Theory and Applications, 30, 287312, 2002.

Cserey, G. and Roska, T.: Immune Response Inspired CNN Algorithms for Many-Target Detection, Proceedings of the 16th European Conference on Circuits Theory and Design ECCTD '03, Kraków, 2003.

Chua, L. O.: Passivity and Complexity, IEEE Trans. on Circuits and Systems: Fundamental Theory and Applications, (CAS-I), 46., 1, 71-81, 1999.

Chua, L.O.: A Paradigm for Complexity World Scientific Series on Nonlinear Science, University of California, Berkeley Series A, 31, 1998.

Chua, L. O. and Roska, T.: The CNN paradigm, IEEE Trans. on Circuits and Systems I, Fundamental Theory and Applications, 40, 147-156, 1993.

Chua, L. O. and Roska, T.: Cellular neural networks ans visual computing, Cambridge University Press ISBN 0-521-65247-2, 8792, 2002.

Chua, L. O. and Roska, T.: Cellular Neural Networks and Visual Computing: Foundations and Applications, Cambridge University Press, 2005.

Chua, L. O. and Yang, L.: Cellular Neural Networks Theory and Applications, IEEE Trans. Circuits Syst., 35, 1257-1290, 1988.

Chua, L. O., Hasler, M., Moschytz, G. S. and Neirynck, J.: Autonomous Cellular Neural Networks: A Unified Paradigm for Pattern Formation and Active Wave Propagation, IEEE Transactions on Circuits and Systems I: Fundamental Theory and Applications, 42, 10, 1995.

Civalleri, P. P., Gilli, M., and Pandolfi, L.: On Stability of Cellular Neural Networks with Delay, IEEE Trans. on Circuits and Systems I: Fundamental Theory and Applications, 40, 157-165, 1993.

Crounse, K. R. and Chua, L. O.: Methods for Image Processing and Pattern Formation in Cellular Neural Networks: A Tutorial, IEEE Trans. on Circuits and Systems I: Fundamental Theory and
Applications, 42, 583-601, 1995a.

Crounse, K. R. and Chua, L. O.: The CNN Universal Machine is as Universal as a Turing Machine, EECS Department, University of California, Berkeley, 1995b.

Cruz, J. M. and Chua, L. O.: A fast Complex and Efficient Testimplementation of the CNN Universal Maschine, Proceedings of the IEEE Int. Workshop on Cellular Neural Networks and their Applications CNNA'94, Rome, 61-66, 1994.

De Souza, S. X., Yalcin, M. E., Suykens, J. A. K., and Vandewalle, J.: Automatic chip-specific CNN template optimization using adaptive simulated annealing, Proceedings of ECCTD'03 conference, Krakow, II., 329-332, 2003.

Di Marco, M., Forti, M., and Tesi, A.: Bifurcations and oscillatory behaviour in a class of competitive cellular neural networks, International Journal of Bifurcation and Chaos, 10, 6, 1267-1294, 2000.

Di Marco, M., Forti, M., and Tesi, A.: Complex dynamics in nearly symmetric three-cell cellular neural networks, International Journal of Bifurcation and Chaos, 12, 6, 1357-1362, 2002.

Domíniguez-Castro, R., Espejo, S., Rodríguez-Váquez, A., and Carmona, R.: A CNN universal chip in CMOS technology, Proceedings of the Third IEEE International Workshop on Cellular Neural Networks and their Applications, 18-21 December 1994, CNNA-94., 18-21 December 1994, Rome, Italy ,ISBN: 0-78032070-0, 91-96, 1994.

Espejo, S., Domíniguez-Castro, R., and Rodríguez-Váquez, A.: Realization of a CNN Universal Chip in CMOS Technology, Proceedings of IEEE International Symposium on Circuits and Systems, 1, 657-659, 1995.

Espejo, S., Rodríguez-Váquez, A., Domíniguez-Castro, R., and Carmona, R.: Convergence and Stability of the FSR CNN Model, Proceedings of the 3rd International Workshop on Cellular Neural Networks and their Applications Rome, 411-416, 1994.

Espejo, S., Rodríguez-Váquez, A., Domíniguez-Castro, R., and Carmona, R.: A VLSI Oriented Continuous-Time CNN Model, International Journal of Circuit Theory and Applications, John Wiley and Sons Ed., 24, 3, 341-356, 1996.

Feiden, D. and Tetzlaff, R.: Obstacle Detection in Planar Worlds using Cellular Neural Networks, Proceedings of the IEEE International Workshop on Cellular Neural Networks and their Applications (CNNA 2002), Frankfurt, 383-390, 2002.

Feiden, D. and Tetzlaff, R.: Coding of Binary Image Data Using Cellular Neural Networks and Iterative Annealing, Proceedings of the European Conference on Circuit Theory and Design ECCTD'03, Krakow, Poland, ECS, ISBN 83-88309-95-1, 229232, 2003.

Gál, V. and Roska, T.: Collision Prediction via the CNN Universal Machine, Proceedings of the IEEE Int. Workshop on Cellular Neural Networks and Their Applications CNNA 2000, Catania, 3-8, 2000.

Geis, G., Reinisch, M., Tetzlaff, R., and Puffer, F.: Linear interpolation of nonlinearities in Cellular Neural Networks, Proceedings of the $8^{\text {th }}$ IEEE International Biannual Workshop of CNNs and their Applications, Budapest, July 2004, 393-398, 2004.

Gilli, M., Roska, T., Chua, L. O., and Civalleri, P. P.: CNN Dynamics Represents a Broader Class than PDEs, International Journal of Bifurcation and Chaos, 12, 2.051-2.068, 2002.

Gollas, F. and Tetzlaff, R.: Modelling Brain Electrical Activity in Epilepsy by Reaction-Diffusion Cellular Neural Networks, Pro- 
ceedings of the Microtechnologies for the New Millenium SPIE 2005 Europe, Sevilla, Spain, 5839, 219-227, 2005a.

Gollas, F. and Tetzlaff, R.: Modelling Complex Systems by Reaction Diffusion Cellular Nonlinear Networks, Proceedings of the IEEE International Workshop on Cellular Neural Networks and their Applications (CNNA 2005), Hsinchu, Taiwan, 227-231, 2005b.

Gollas, F., Niederhöfer, C., and Tetzlaff, R.: Prediction of Brain Electrical Activity in Epilepsy Using a Higher-Dimensional Prediction Algorithm for Discrete Time Cellular Neural Networks (DTCNN), Proceedings of the IEEE International Symposium on Circuits and Systems ISCAS, Vancouver, 2004.

Joy, M. P. and Tavsanoglu, V.: An Equilibrium Analysis of CNN's, IEEE Trans. on Circuits and Systems I: Fundamental Theory and Applications, 45, 94-98, 1998.

Kim, H., Park, Y., Roska, T., and Chua, L. O.: Optimal Path Finding with the Nonlinear Templates of Multi-Layer CNN, Proceedings of the IEEE Workshop on Signal Processing Systems (SiPS 2000): Design and Implementation, 455-460, 2000.

Koskinen, L., Laiho, M., Paasio, A., and Halonen, K.:MPEG-4 based modifications for an CNN segmentation chip, Proceedings of the 2002 7th IEEE International Workshop on Cellular Neural Networks and Their Applications, 2002 (CNNA 2002), 22-24 July 2002, ISBN: 981-238-121-X, 71-77, 2002.

Liñán, G., Domíniguez-Castro, R., Espejo, S., and RodriguezVázquez, A.: Design of a Large-Complexity Analog I/O CNNUC, Design Automation Day proceedings, European Conference on Circuit Theory and Design, ECCTD'99, 40, 42-57, 1999a.

Liñán, G., Domínguez-Castro, R., Espejo, S., and RodríguezVáquez, A.: Design of large complexity analog I/O CNNUC, Proceedings of the 14th European Conference on Circuit Theory and Design -ECCTD 99, Stresa, Italy, 1999b.

Liñán, G., Espejo, S., Domíniguez-Castro, R., and RodríguezVáquez, A.: ACE4k: an analog I/O 64 x 64 visual microprocessor chip with 7-bit analog accuracy, International Journal of Circuits and Applications 2002, John Wiley and Sons LTD., 30, 89-116, 2002.

Loncar, A. and Tetzlaff, R.: Cellular Neural Networks with Nearly Arbitrary Nonlinear Weight Functions, Proceedings of IEEE Int. Workshop on Cellular Neural Networks and Their Applications, 171-176, 2000.

Martínez, J., Toledo, F. J., and Ferrández, J. M.: Implementation of a discrete cellular neuron model (DT-CNN) architecture on FPGA, Proceedings of the SPIE Bioengineered and Bioinspired Systems II. Edited by Carmona, Ricardo A.; Liñán-Cembrano, Gustavo, 5839, 332-340, 2005.

McRaven, J., Scheutz, M., Cserey, G., Andronache, V., and Porod, W.: Fast Detection and Tracking of Faces in Uncontrolled Environments for Autonomous Robots Using the CNN-UM, Proceedings of the The 8th IEEE International Biannual Workshop on Cellular Neural Networks and their Applications CNNA '04, Budapest, Hungary 2004.

Murray, J. D.: Mathematical Biology, Springer Verlag, 1989.

Nagy, Z. and Szolgay, P.: Configurable Multi-Layer CNN-UM Emulator on FPGA, IEEE Transactions on CAS I, 50, 774-778, 2003.

Puffer, F., Tetzlaff, R., and Wolf, D.: Modeling nonlinear systems with Cellular Neural Networks, Proceedings of the IEEE Interna- tional Conference On Acoustics, Speech And Signal Processing 1996, ICASSP 1996, Atlanta, USA, 3513-3516, 1996 a.

Puffer, F., Tetzlaff, R., and Wolf, D.: A Learning Algorithm for the Dynamics with Nonlinear Templates-Part II: Continuous-Time Case, Proceedings of IEEE Int. Workshop on Cellular Neural Networks and Their Applications, 467-472, $1996 \mathrm{~b}$.

Reinisch, M., Geis, G., and Tetzlaff, R.: System Identification by Cellular Neural Networks (CNN): Linear Interpolation of Nonlinear Weight Functions, Proceedings of the Microtechnologies for the New Millenium SPIE Europe, 9-11 May 2005, Seville, Spain, 353-358, 2005.

Rekeczky, C., Tahy, Á., Végh, Z. and Roska, T.: Spatio-temporal Nonlinear Filtering and Endocardial Boundary Detection in Echocardiography, Proceedings of the 13th IEEE European Conference on Circuit Theory and Design ECCTD '97, Budapest, August 1997, 677-672, 1997.

Rekeczky, C., Roska, B., Nemeth, E., and Werblin, F. S.: Neuromorphic CNN Models for Spatio-Temporal Effects Measured in the Inner and Outer Retina of Tiger Salamander, Proceedings of IEEE Int. Workshop on Cellular Neural Networks and Their Applications, 15-20, 2000.

Rodríguez-Váquez, a., Espejo, s., Domíniguez-Castro, R., Huertas, J. L., and Sánchez-Sinencio E.: Analog and Digital Signal Processing, IEEE Transactions on Circuits and Systems II, 40, 132, 1993.

Roska, T. and Chua, L. O.: Cellular Neural Networks with nonlinear and delay-type template elements and non-uniform grids, Int. Journal of Circuit Theory and Applications, 20, 469-481, 1992.

Roska, T. and Chua, L. O.: The CNN universal machine: an analogic array computer, IEEE Transactions on Circuits and Systems II:Analog and Digital Processing, March 1993, 40, 163173, 1993.

Saatci, E. and Tavsanoglu, V.: Fingerprint image enhancement using CNN Gabor-Type filters, Proceedings of the 2002 7th IEEE International Workshop on Cellular Neural Networks and Their Applications, 2002 (CNNA 2002), 22-24 July 2002, ISBN: 981-238121-X, 377-382, 2002.

Slavik, Z., Tetzlaff, R., Blug, A., and Höfler, H.: Visual inspection of metal objects by using Cellular Neural Networks, Proceedings of the 10th International Workshop on Cellular Neural Networks and Their Applications CNNA, 28-30 August 2006, Istanbul, Türkei, 142-146, 2006.

Suykens, J. A., Munuzuri, A. P., Vandewalle, J., and Chua, L. O.: Phenomena from CNNs with generalized Chua's Circuits, Proceedings of International Symposium on Nonlinear Theory and Applications, 1, 205-208, 1997.

Tetzlaff, R.: Solitons in Cellular Nonlinear Networks, Proceedings of the European Conference on Circuit Theory and Design ECCTD'03, Krakau, Polen, ECS, ISBN 83-88309-95-1, 434439, 2003.

Tetzlaff, R. and Kunz, R.: Spatio-Temporal Distibution of Brain Electrical Activity Patterns in Epilepsy: Inputs for Cellular Neural Networks, Proceedings of the IEEE International Symposium on Circuits and Systems (ISCAS'2001), Sydney, 3, 329332, 2001.

Kunz, R. and Tetzlaff, R.: Spatio-Temporal Dynamics of Brain Electrical Activity in Epilepsy: Analysis with Cellular Neural Networks (CNNs), Journal of Circuits, Systems, and Computers, 
12, 825-844, 2003.

Tetzlaff, R., Kunz, R., and Geis, G.: Analysis of Cellular Neural Networks with Parameter Deviations, Proceedings of the European Conference on Circuit Theory and Design, Budapest, 650654, 1997.

Tetzlaff, R., Kunz, R., and Wolf, D.: Minimizing the Effects of Parameter Deviations on Cellular Neural Networks, Int. Journal of Circuit Theory and Applications, John Wiley and Sons, 27, 1, 77-87, 1999a.

Tetzlaff, R., Kunz, R., Ames, C., and Wolf, D.: Analysis of Brain Electrical Activity in Epilepsy with Cellular Neural Networks, Proceedings of the European Conference on Circuit Theory and Design, Stresa, 1007-1010, 1999b.

Tetzlaff, R., Niederhöfer, C., and Fischer, P.: Automated Detection of a Preseizure State: Nonlinear EEG Analysis in Epilepsy by Cellular Nonlinear Networks and Volterra Systems, International Journal of Circuit Theory and Applications, 2005.
Toledo, F. J., Martínez, J. J., Garrigos, F. J., Ferrández, J. M., Cabestany, J., Prieto, A., and Sandoval F.: Image processing with CNN in a FPGA-based augmented reality system for visually impaired people, Proceedings of the Computational intelligence and bioinspired systems (Barcelona, 8-10 June 2005) [Note(s): XXV, 1260 p.] (13 ref.), 3512, 906-912, 2005.

Vilarino, D. and Rekeczky, C.: Shortest Path Problem with Pixel Level Snakes: Application to Robot Path Planning, Proceedings of the The 8th IEEE International Biannual Workshop on Cellular Neural Networks and their Applications CNNA '04, Budapest, Hungary 2004.

Werblin, F. S. and Roska, B. M.: Parallel visual processing, A tutorial of retinal function, International Journal of Bifurcation and Chaos, World Scientific Publishing Company, 14, 843-852, 2004.

Zarandy, A. and Rekeczky, C.: Bi-i: A standalone ultra high speed cellular vision system, IEEE Circuits and Systems Magazine, 5, 36-45, 2005. 